\title{
Efeito do pareamento ao modelo com atraso fixo ou ajustado na formação de classes de equivalência*
}

\section{Effect of matching to sample with fixed or adjusted delay on equivalence class formation
Efecto del emparejamiento con modelo de retraso fijo o ajustado en la formación de clases de equivalencia

\section{Camila Fernanda Soares Leal ${ }^{1}$, Raquel Maria de Melo $^{2}$}

[1] [2] Universidade de Brasília I Título abreviado: Pareamento com atraso e equivalência I Endereço para correspondência: Camila Fernanda Soares Leal Departamento de Processos Psicológicos Básicos/Instituto de Psicologia, Universidade de Brasília - Campus Universitário Darci Ribeiro; Brasília/DF; CEP: 70.910900 - Telefone: (61) 3107-2844; FAX: (61) 3273-0203 I Email: camilafs|@outlook.com I doi.org/10.18761/PAC.2020.v11.n2.05

Resumo: O presente estudo teve como objetivo investigar o efeito de diferentes tipos de pareamento ao modelo com atraso na formação de classes de equivalência com um delineamento intrassujeito. Cinco estudantes universitários foram expostos a duas condições experimentais. Na Condição Atraso Ajustado, o atraso entre a apresentação dos estímulos modelo e de comparação nos treinos aumentava de $1 \mathrm{em} 1 \mathrm{~s}$ até atingir o atraso máximo de 12 s e na Condição Atraso Fixo o atraso era sempre de $12 \mathrm{~s}$. O número de tentativas foi acoplado entre as condições experimentais. Após o treino de duas discriminações condicionais (AB e $\mathrm{AC}$ ou DE e DF), com estímulos visuais abstratos, foram realizados os testes de simetria, transitividade e equivalência. Os resultados demonstram que na Condição Atraso Fixo todos os participantes formaram classes de equivalência enquanto na Condição Atraso Ajustado apenas três. Esses resultados corroboram com a literatura ao demonstrarem que melhores desempenhos são obtidos após o treino de relações condicionais com pareamento com atraso fixo quando comparado aos desempenhos obtidos após o treino de relações condicionais com pareamento com atraso ajustado.

Palavras-chave: pareamento ao modelo, atraso fixo, atraso ajustado, equivalência de estímulos 
Abstract: The present study aimed to investigate the effect of different types of matching to sample with delay in the formation of equivalence classes with an intrasubject design. Five undergraduate students were exposed to two experimental conditions. In the Adjusted Delay Condition, the delay between the presentation of model and comparison stimuli in training increased from 1 to $1 \mathrm{~s}$ until reaching the maximum delay of $12 \mathrm{~s}$ and in the Fixed Delay Condition the delay was always $12 \mathrm{~s}$. The number of trials was coupled between the experimental conditions. After the training of two conditional discriminations $(\mathrm{AB}$ and $\mathrm{AC}$ or DE and DF), with abstract visual stimuli, symmetry, transitivity and equivalence tests were performed. Results show that in Fixed Delay Condition all participants formed equivalence classes while in Adjusted Delay Condition only three. These results corroborate the literature by demonstrating that better performances are obtained after the conditional relations training with fixed delayed pairing when compared to the performances obtained after the training of conditional relations with adjusted delayed pairing.

Keywords: matching to sample, fixed delay, adjusted delay, stimulus equivalence

Resumen: El presente estudio tuvo como objetivo investigar el efecto de diferentes tipos de igualación con la muestra con retraso en la formación de clases de equivalencia con un diseño intra-sujeto. Cinco estudiantes universitarios fueron expuestos a dos condiciones experimentales. En la Condición de retraso ajustada, la demora entre la presentación del modelo y los estímulos de comparación en el entrenamiento aumentó de 1 a $1 \mathrm{~s}$ hasta alcanzar el retraso máximo de $12 \mathrm{~s}$ y en la Condición de retraso fijo el retraso fue siempre de $12 \mathrm{~s}$. El número de ensayos se acopló entre las condiciones experimentales. Después del entrenamiento de dos discriminaciones condicionales ( $\mathrm{AB}$ y AC, Condición de retraso ajustada, y DE y DF, Condición de retraso fijo), con estímulos visuales abstractos, se realizaron pruebas de simetría, transitividad y equivalencia. Los resultados muestran que en condición de retraso fijo todos los participantes formaron clases de equivalencia, mientras que en condición de retraso ajustado solo tres. Estos resultados corroboran la literatura al demostrar que se obtienen mejores rendimientos después del entrenamiento de relaciones condicionales con emparejamiento retrasado fijo en comparación con los desempeños obtenidos después del entrenamiento de relaciones condicionales con emparejamiento retrasado ajustado.

Palabras clave: igualación con la muestra, retraso fijo, retrasado ajustado, relaciones de equivalencia

* Nota dos Autores: O estudo é parte da dissertação de mestrado da primeira autora sob orientação da segunda autora, desenvolvido no Programa de Pós-Graduação em Ciências do Comportamento do Departamento de Processos Psicológicos Básicos da Universidade de Brasília. Durante a realização do estudo, a primeira autora era bolsista da CAPES.

Esse estudo está inserido no programa de pesquisa do Instituto Nacional de Ciência e Tecnologia (INCT-ECCE), com apoio financeiro do CNPq (Processo 573972/2008-7) e da FAPESP (Processo 2008/57705-8).

Declara-se que a presente pesquisa está em conformidade com os princípios éticos e foi aprovada pelo Comitê de Ética em Pesquisa em Ciências Humanas e Sociais - CEP/CHS, com parecer: 3.235.295. 
O paradigma de equivalência de estímulos tem sido utilizado na Análise do Comportamento para operacionalizar a ocorrência de comportamentos novos, complexos e simbólicos, como os que estão envolvidos na leitura, na escrita, e na aprendizagem de conceitos e de habilidades matemáticas (Albuquerque \& Melo, 2005; Sidman \& Tailby, 1982). Tais comportamentos podem emergir, ou seja, serem aprendidos sem a necessidade de um treino direto, a partir do ensino de relações condicionais com estímulos que passam a compartilhar uma função entre si, tornando-se substituíveis no controle do comportamento (Albuquerque \& Melo, 2005).

O estudo de comportamentos emergentes a partir do referencial da equivalência de estímulos se dá por investigação de variáveis que afetam a formação de classes equivalência. Inicialmente, são realizados treinos de discriminações condicionais (e.g., $\mathrm{AB}$ e $\mathrm{AC}$ ), geralmente, com o procedimento de pareamento ao modelo (matching to sample, em inglês). Posteriormente, a formação de classes de equivalência é verificada por relações emergentes que atestam as propriedades de reflexividade (relação de identidade na qual $\mathrm{A}=\mathrm{A}, \mathrm{B}=\mathrm{B}$ e $\mathrm{C}=\mathrm{C}$ ), simetria (se $A=B$, então $B=A$ ), transitividade (se $A$ $=\mathrm{B}$ e $\mathrm{B}=\mathrm{C}$, então $\mathrm{A}=\mathrm{C}$ ) e equivalência (se $\mathrm{A}=\mathrm{C}$, então $C=A$ ). Tais propriedades atestam novas relações entre estímulos, ou seja, relações diferentes das relações previamente treinadas, e o compartilhamento de uma função em comum dos estímulos no controle do comportamento (Albuquerque \& Melo, 2005; Sidman \& Tailby, 1982; Sidman, Willson-Morris, \& Kirk, 1986).

O tipo de procedimento de treino de pareamento ao modelo é uma das variáveis que pode afetar a formação de classe de equivalência. Dentre as tarefas de pareamento ao modelo podem ser caracterizados: (1) o pareamento ao modelo com apresentação simultânea dos estímulos modelo e de comparação (do inglês, Simultaneous Matching to Sample - SMTS). Neste procedimento, após a ocorrência de uma resposta de observação ao estímulo modelo, os estímulos de comparação são apresentados e permanecem disponíveis durante toda a tentativa simultaneamente com o estímulo modelo; e (2) o pareamento ao modelo com atraso (do inglês, Delayed Matching to Sample - DMTS). Nesta tarefa, a resposta de observação ao estímulo modelo resulta na sua remoção e, após um determinado período, definido pelo experimentador, são apresentados os estímulos de comparação. Assim, a resposta de seleção de um dos estímulos de comparação ocorre na ausência do estímulo modelo. O tempo decorrido entre a remoção do estímulo modelo e a apresentação dos estímulos de comparação, ou atraso, pode ser fixo ou ajustado. No pareamento com atraso fixo (do inglês, Fixed Delayed Matching to Sample - FDMTS) o tempo decorrido entre a remoção do estímulo modelo e a apresentação dos estímulos de comparação é sempre o mesmo. No procedimento de pareamento com atraso ajustado (do inglês, Titrating Delayed Matching to Sample - TDMTS) o atraso muda durante o treino, sendo ajustado gradualmente de acordo com o desempenho do participante (Arntzen, 2012; Ferraro, Francis, \& Perkins, 1971).

Nos estudos que utilizaram o procedimento de pareamento simultâneo, a formação de classes de equivalência tem sido relatada com crianças (e.g., de Rose, de Sousa, \& Hanna, 1996; Melo \& Serejo, 2009), adultos (e.g., Laporte \& Melo, 2016; Wulfert \& Hayes, 1988), idosos (e.g., Aggio \& Domeniconi, 2012; Haydu \& Morais, 2009) e pessoas com comprometimento neurológico (e.g., Carr, Wilkinson, Blackman, \& McIlvalne, 2000; Sidman \& Cresson, 1973). Com relação ao procedimento com atraso, verifica-se na literatura que: (1) o pareamento com atraso favorece a emergência de relações indiretamente treinadas quando comparado com o pareamento simultâneo (e.g., Bortoloti \& de Rose, 2012; Saunders, Chaney, \& Marquis, 2005); e (2) maiores atrasos tendem a produzir melhores resultados no teste de relações emergentes de simetria (e.g., Vaidya \& Smith, 2006) e equivalência (e.g., Arntzen, 2006; Lian \& Arntzen, 2013). De acordo com Arntzen (2006), o pareamento com atraso permite investigar: (1) propriedades de controle de estímulos em situações em que um dos estímulos, condicional ou discriminativo, não está presente quando o indivíduo emite a resposta; (2) comportamentos precorrentes de natureza privada, como nomeação e ensaio, que podem ocorrer durante a execução da tarefa de pareamento; e (3) o efeito da duração do atraso entre a remoção do estímulo modelo e a apresentação dos estímulos de comparação sobre o responder emergente. 
Arntzen (2006) investigou, em três experimentos, o efeito da duração do atraso na formação de classes de equivalência com estímulos visuais abstratos (e.g., letras gregas e árabes). No Experimento 1, 24 participantes foram expostos a duas ordens dos atrasos: crescente (simultâneo, $0 \mathrm{~s}, 2 \mathrm{~s}$ e $4 \mathrm{~s}$ ) e decrescente ( $4 \mathrm{~s}, 2 \mathrm{~s}, 0$ s e simultâneo). Nos testes de formação de classes foi utilizado o procedimento de pareamento simultâneo. Dentre os 12 participantes expostos à ordem crescente dos atrasos, sete apresentaram desempenhos nos testes que evidenciaram a formação de classes de equivalência com o pareamento simultâneo; 10 quando o atraso foi $0 \mathrm{~s}$; 11 quando o atraso foi $2 \mathrm{~s}$; e todos os participantes quando o atraso foi 4s. Com relação a exposição à ordem decrescente dos atrasos, foi verificado que 10 participantes formaram classes de equivalência quando o atraso foi $4 \mathrm{~s} ; 11$ quando o atraso foi $0 \mathrm{~s}$; e todos os 12 participantes com o pareamento simultâneo e o atraso de $2 \mathrm{~s}$. Esses resultados, no entanto, não permitem concluir se a formação das classes de equivalência foi controlada pela duração dos atrasos ou pela ordem de exposição aos atrasos, uma vez que foi verificada para todos os participantes no último atraso ( $4 \mathrm{~s}$ - ordem crescente e simultâneo ordem decrescente). No Experimento 2, seis participantes foram expostos apenas à ordem crescente dos atrasos. Independente do atraso, todos os participantes responderam de acordo com o critério de formação de classes de equivalência. $\mathrm{O}$ atraso de $0 \mathrm{~s}$ foi o que requereu um maior número de tentativas nos treinos para que o critério fosse atingido. No Experimento 3, os atrasos de $2 \mathrm{~s}$ e $4 \mathrm{~s}$ foram substituídos por $3 \mathrm{~s}$ e $9 \mathrm{~s}$, respectivamente. Todos os participantes formaram classes de equivalência e, assim como no Experimento 2, com o atraso de 0 $\mathrm{s}$ foi necessário um maior número de tentativas de treino, seguido do atraso de $9 \mathrm{~s}$, e o menor número ocorreu para o atraso de $3 \mathrm{~s}$.

O estudo de Arntzen (2006) investigou o efeito de diferentes durações do atraso na formação de classes de equivalência, porém não foram apresentados os resultados intrassujeito, o que dificulta o mapeamento do desempenho individual em cada atraso. Além disso, com relação aos treinos de discriminação condicional nos Experimentos 2 e 3, o atraso $0 \mathrm{~s}$ requereu um maior número de tentativas em comparação aos demais atrasos (exceto o atra- so de 9 s, utilizado apenas no Experimento 3), e à apresentação simultânea dos estímulos.

O estudo de Arntzen, Galaen e Halvorsen (2007) teve como objetivo ampliar as investigações de Arntzen (2006) acerca do efeito de diferentes atrasos sobre a formação de classes de equivalência, com estímulos visuais abstratos. Vinte adultos, com idades entre 20 e 45 anos, foram expostos a treinos de pareamento ao modelo com atrasos de $0 \mathrm{~s}, 6 \mathrm{~s}$ e $12 \mathrm{~s}$. A ordem de exposição aos atrasos (crescente ou decrescente) foi contrabalanceada entre os participantes. Foi utilizado o procedimento de pareamento simultâneo nas tentativas de teste. Nove participantes expostos à ordem crescente atingiram o critério de formação de classes de equivalência com atraso $0 \mathrm{~s}$ e todos os 10 participantes atingiram o critério nos demais atrasos. Nove dos participantes expostos à ordem decrescente formaram classes de equivalência com os atrasos $12 \mathrm{~s} \mathrm{e} 6 \mathrm{~s}$, e todos os 10 com o atraso $0 \mathrm{~s}$. O número de tentativas e de erros nos treinos foi maior durante o primeiro atraso, independentemente da duração ( $0 \mathrm{~s}$ ou $12 \mathrm{~s}$ ).

Os resultados obtidos por Arntzen et al. (2007) demonstram um efeito de ordem importante, uma vez que o maior número de erros e de tentativas nos treinos ocorreu na primeira exposição à tarefa, independentemente da duração do atraso, e diminuiu à medida que as exposições à tarefa de pareamento ao modelo com atraso foram realizadas. No estudo de Arntzen (2006) foi verificado o mesmo padrão para a ordem crescente dos atrasos, porém os desempenhos com a ordem decrescente dos atrasos apresentaram pouca sistematicidade, uma vez que o número de participantes que formou classes de equivalência não variou de acordo com o tamanho do atraso e nem de acordo com a exposição à tarefa. Além disso, os resultados de Arntzen et al. (2007) contrastam com os que foram obtidos nos Experimentos 2 e 3 de Arntzen (2006) em relação à ordem crescente dos atrasos, pois o número de erros e de tentativas de treino podem ter variado em função da ordem de exposição à tarefa e não apenas em função da duração do atraso. Assim como em Arntzen (2006), Arntzen et al. (2007) não apresentaram os dados individuais, o que dificulta a análise intrassujeito do efeito da duração do atraso sobre a formação de classes de equivalência.

Arntzen (2006) e Arntzen et al. (2007) utili- 
zaram apenas atrasos fixos durante os treinos. No entanto, como foi mencionado anteriormente, o procedimento de pareamento com atraso ajustado vem sendo utilizado em estudos sobre treinos de discriminação condicional para investigar se o aumento gradual da duração do atraso, em função do comportamento do indivíduo, favorece a ocorrência de desempenhos emergentes (Arntzen \& Steingrimsdottir, 2014; Lian \& Arntzen, 2011).

Arntzen, Steingrimsdottir e Antonsen (2013) realizaram dois experimentos, com uma participante com 85 anos, diagnosticada com Alzheimer, para investigar o efeito do atraso no desempenho em uma tarefa de pareamento de identidade com estímulos familiares. No Experimento 1, a participante foi exposta a um delineamento $\mathrm{ABA}$ em que nas duas Condições A foi utilizado o atraso fixo de $12 \mathrm{~s}$ e na Condição B o atraso fixo de $10 \mathrm{~s}$. Foi verificado responder de acordo com o pareamento de identidade apenas quando o atraso era de $10 \mathrm{~s}$. O Experimento 2, realizado com o procedimento de pareamento com atraso ajustado, investigou parametricamente qual a duração máxima do atraso que produziria o responder relacional de identidade. A participante foi exposta a sete condições. Nas Condições 1 e 2 o atraso inicial foi $10 \mathrm{~s}$. As Condições 2 a 7 variaram com base na duração de apresentação das consequências (1 s nas Condições 4 e 6; 3 s na Condição 7; e 1,5 s para as demais condições) e do intervalo entre tentativas - IET $(0 \mathrm{~s}$ nas Condições 3 e 4 e 0,5 s nas demais condições). $\mathrm{O}$ atraso inicial das Condições 3 a 7 foi definido a partir do atraso final da condição anterior. $\mathrm{O}$ atraso foi ajustado em $0,25 \mathrm{~s}$ entre os blocos de tentativas, conforme acerto ou erro. O critério estabelecido para considerar que a duração do atraso não mais produziria efeito sobre o responder por identidade foi a realização de cinco blocos consecutivos no qual o valor do atraso não avançasse. Foi verificado responder por identidade mesmo quando o atraso foi de 12,25 s (Condição 3), duração superior ao programado durante as Condições A do Experimento 1.

No estudo de Lian e Arntzen (2011), composto por três experimentos, crianças entre seis e 10 anos foram expostas a treinos com o procedimento de pareamento com diferentes atrasos. Vinte crianças participaram do Experimento 1, sendo que metade foi exposta a $3 \mathrm{~s}$ de atraso e a outra metade a $6 \mathrm{~s}$ de atraso durante o treino com estímulos visuais abstratos. Os participantes do Grupo $6 \mathrm{~s}$ atingiram o critério de acurácia nos treinos de discriminações condicionais com um número médio de tentativas superior ao do Grupo 3 s. Nos testes, com o procedimento de pareamento simultâneo, foi verificado que 15 participantes, oito do Grupo 3 s e sete do Grupo 6 s, formaram classes de equivalência. Esses resultados mostram que, com crianças, atrasos mais longos não favoreceram a formação de classes de equivalências em relação a atrasos mais curtos. No Experimento 2, participaram 20 crianças organizadas em dois grupos, 3 s e $6 \mathrm{~s}$ de atrasos ajustados. $\mathrm{O}$ atraso iniciava em $0 \mathrm{~s}$ e aumentava $250 \mathrm{~ms}$ em cada bloco de tentativas para o Grupo 3 s e $500 \mathrm{~ms}$ para o Grupo 6 s; em caso de erros, o atraso era reduzido nesses mesmos valores. Para verificar apenas o efeito do tipo do atraso, outros oito participantes foram expostos a uma Condição Controle, sendo que quatro realizaram treinos com atraso fixo de $100 \mathrm{~ms}$ e quatro com $100 \mathrm{~ms}$ de atraso ajustado (o atraso iniciava e $0 \mathrm{~s}$ e avançava ou reduzia em $8 \mathrm{~ms}$, dependendo do desempenho do participante). Os participantes expostos ao atraso de $6 \mathrm{~s}$ ajustado necessitaram de mais tentativas para atingir o critério nos treinos do que os participantes expostos a $3 \mathrm{~s}$ de atraso ajustado, o que corrobora com os resultados do Experimento 1. Entretanto, para os participantes da Condição Controle não foi verificada diferença na formação de classe de equivalência após treinos com atrasos fixo e ajustado. No Experimento 3, realizado com 10 crianças, $\mathrm{o}$ atraso inicial foi alterado de $0 \mathrm{~s}$ para 1,5 s para investigar se a quantidade de erros, observada no Experimento 2, seria reduzida. $\mathrm{O}$ atraso máximo utilizado era de $3 \mathrm{~s}$ e a partir de $1,5 \mathrm{~s}$, aumentava gradualmente em $125 \mathrm{~ms}$. Foi verificado que a o atraso de $1,5 \mathrm{~s}$ resultou em diminuição no número de erros em relação ao atraso de $0 \mathrm{~s}$, mas apenas em um dos treinos.

Os resultados dos estudos de Lian e Arntzen (2011) e Arntzen et al. (2013) sugerem que desempenhos distintos podem ser obtidos a partir de diferentes manipulações do atraso. Lian e Arntzen (2011) utilizaram um delineamento entre sujeitos, o que impede a análise do desempenho individual e a comparação controlada dos efeitos do tipo de manipulação do atraso, se fixo ou ajustado, sobre a formação 
de classes de equivalência. Outra limitação, observada e discutida pelos autores, refere-se à ausência de controle do número de tentativas nos dois experimentos, uma vez que a exposição maior ou menor às tentativas de treino pode afetar o desempenho nos testes de relações emergentes. No estudo de Arntzen et al. (2013) foi utilizado o delineamento intrassujeito e os resultados divergem dos obtidos por Lian e Arntzen (2011), uma vez que demonstram melhores desempenhos nos testes após o treino com pareamento com atraso ajustado. Nesses estudos também foi manipulado o atraso, fixo ou ajustado, dentre as tentativas de teste e não apenas durante as tentativas de treino, como em Arntzen (2006), Arntzen et al. (2007) e Lian e Arntzen (2011). Entretanto, os autores utilizaram apenas a tarefa de pareamento de identidade, o que não permite verificar a formação de classes de equivalência.

Considerando as limitações dos delineamentos e do controle experimental observadas nos estudos de Arntzen (2006), Arntzen, et al. (2007) e Lian e Arntzen (2011), o presente estudo teve como objetivo investigar o efeito dos diferentes tipos de pareamento ao modelo com atraso, fixo ou ajustado, na formação de classes de equivalência com um delineamento intrassujeito e com controle do número de tentativas nos treinos com cada tipo de atraso.

\section{Método}

\section{Participantes}

Participaram, voluntariamente, do estudo cinco estudantes de graduação da Universidade de Brasília, sendo duas mulheres e três homens, com idades entre 19 e 25 anos, e procedentes de cursos de diferentes áreas do conhecimento. Todos os estudantes atenderam aos seguintes critérios: não ser aluno do curso de Psicologia e não possuir história anterior de participação em pesquisas que utilizaram o procedimento de pareamento ao modelo.

A presente pesquisa foi aprovada pelo Comitê de Ética em Pesquisa [informação suprimida para não identificar o(s) autor(es)] - CAAE: 08809319.0.0000.5540, com Parecer: 3.235.295. Antes de iniciar as tarefas, todos os participantes leram e assinaram o Termo de Consentimento Livre e Esclarecido (TCLE).

\section{Local e materiais}

Foi utilizada uma das salas de coleta de dados do Laboratório Integrado de Pós-Graduação e Pesquisa Experimental [informação suprimida para não identificar o(s) autor(es)]. A sala utilizada mede aproximadamente $6 \mathrm{~m}^{2}$, possui iluminação artificial e sistema de ar-condicionado, e foi equipada com duas mesas e duas cadeiras.

Para a realização das tarefas experimentais foi utilizado um microcomputador, com mouse, e o software Contingência Programada versão 2.0 (Hanna, Batitucci, \& Batitucci, 2014) para a programação das tarefas de pareamento ao modelo. Esse software permite a apresentação de estímulos auditivos e visuais, consequências para acertos e erros, e registro de respostas de seleção.

\section{Estímulos}

Foram utilizados 66 estímulos visuais, organizados em seis conjuntos, que consistiam em símbolos abstratos, procedentes dos estudos de Nalini (2002), Oliveira (2014), Wilson e Hayes (1996), Garcia e Rehfeldt (2008), e de buscas na internet a partir dos descritores "símbolos abstratos" e "línguas mortas". Os estímulos dos conjuntos A e D continham apenas um elemento e foram utilizados apenas com a função de estímulos modelo nas tarefas de pareamento ao modelo. Os estímulos dos conjuntos B e C (Condição Atraso Ajustado) e E e F (Condição Atraso Fixo) eram compostos por dois elementos e foram utilizados com a função de estímulos de comparação.

Para cada estímulo de comparação definido como correto $(\mathrm{S}+)$ nas tarefas de pareamento ao modelo, quatro estímulos incorretos (S-) foram confeccionados de acordo com os seguintes critérios: (1) o primeiro S- foi composto por um símbolo igual ao $\mathrm{S}+$ e na mesma posição (esquerda ou direita), e um elemento diferente; (2) o segundo $\mathrm{S}$ - foi composto por um símbolo igual ao $\mathrm{S}+$, mas em uma posição diferente, e um símbolo diferente; (3) o terceiro S- foi composto por um símbolo igual a um dos elementos utilizados no primeiro ou no segundo S-, mas diferente dos símbolos que compunham o S+, e um símbolo diferente; e (4) o quarto estímulo foi composto por dois símbolos completamente diferentes dos elementos utilizados nos outros três S- (Figura 1). 


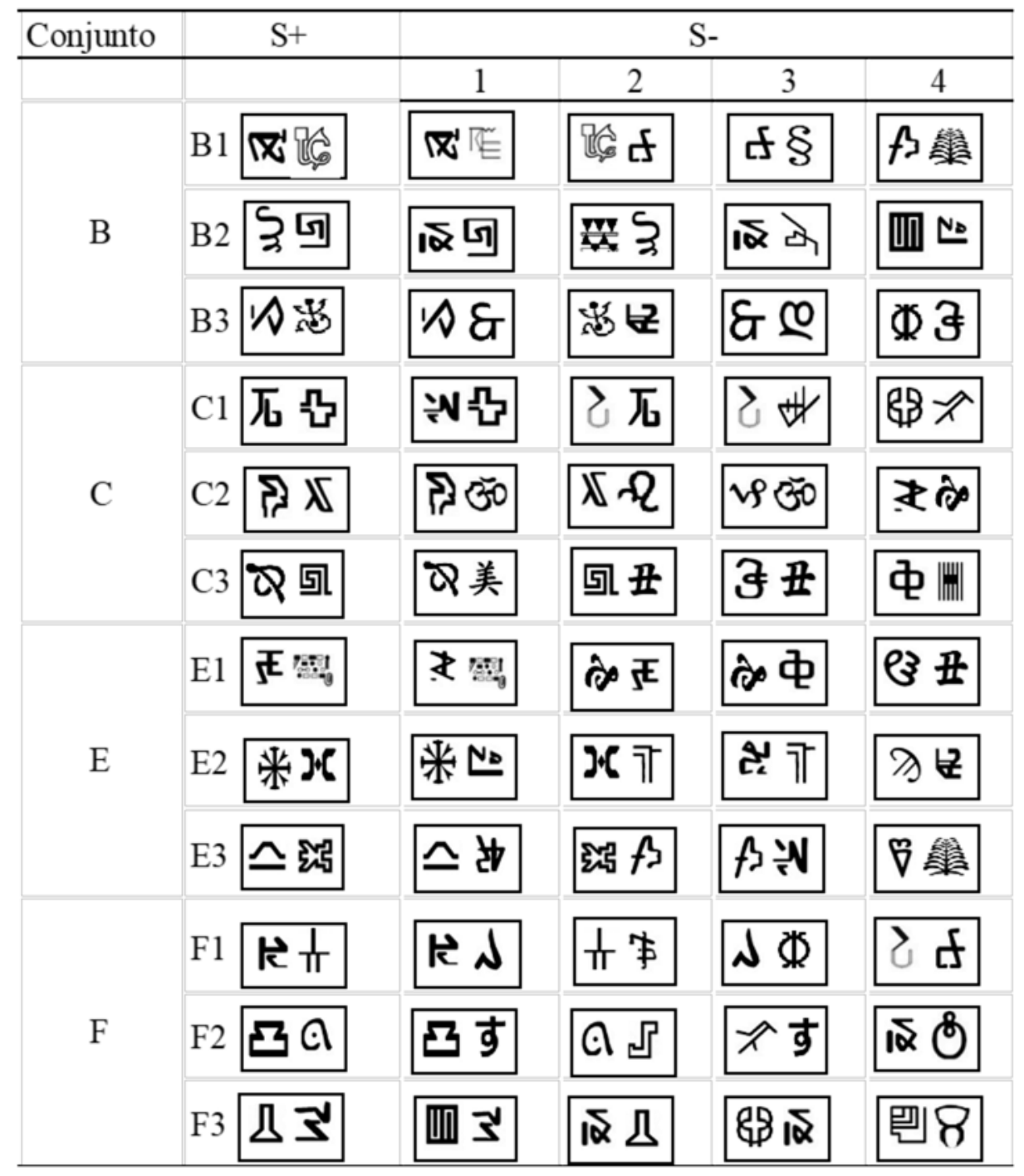

Figura 1. Conjuntos com três estímulos das duas condições experimentais, com a identificação dos estímulos utilizados como S- para cada S+.

\section{Procedimento}

Foi utilizado o delineamento intrassujeito. Cada participante foi exposto a duas condições experimentais, com estímulos distintos, que se diferenciavam pelo tipo de atraso manipulado nas etapas de treino com o procedimento de pareamento ao modelo com atraso: Condição Atraso Ajustado e Condição Atraso Fixo. Os participantes foram expostos primeiramente à Condição Atraso Ajustado e, com base no número de blocos de tentativas realizados nesta condição, a Condição Atraso Fixo foi programada. A escolha desse delineamento permi- tiu que o número de blocos de tentativas fosse mantido constante nas duas condições.

Cada condição era composta por treinos simples de duas relações condicionais $(\mathrm{AB}$ e $\mathrm{AC}$ Condição Atraso Ajustado ou DE e DF - Condição Atraso Fixo), seguidos do treino misto, com tentativas misturadas dessas duas relações, e testes de formação de classe de equivalência que avaliava a emergência das relações de simetria, transitividade e equivalência (Tabela 1). Os treinos das relações condicionais foram realizados de acordo com a es- 
trutura de treino Um-para-Muitos, na qual apenas um conjunto de estímulos era apresentado como modelo (Conjunto A ou Conjunto D) e os demais conjuntos eram apresentados como estímulos de comparação (B e C ou E e F). Os treinos e testes das duas condições experimentais foram realizados em duas sessões de, aproximadamente, uma hora de duração.

Treino de relações condicionais da Condição Atraso Ajustado. Cada treino simples (AB e AC) era composto por 12 blocos, com três tentativas. No primeiro bloco de cada treino, eram apresentadas as dicas visuais (sentenças escritas) "Na presença deste", acima do estímulo modelo e "Escolha este", localizada acima do estímulo de comparação correspondente ao modelo $(\mathrm{S}+)$. Estas dicas foram removidas no segundo bloco de tentativas.

Cada tentativa de pareamento com atraso ajustado iniciava com o estímulo modelo na parte superior da tela e o participante deveria clicar sobre esse estímulo, o que foi definido como resposta de observação. A seguir, o estímulo modelo era removido e os três estímulos de comparação (o S+ e dois S-, selecionados randomicamente dentre quatro possibilidades, conforme Tabela 1) eram apresentados na parte inferior da tela após atrasos ajustados gradualmente, de 1 em $1 \mathrm{~s}$ a partir do atraso inicial de $1 \mathrm{~s}$, a cada bloco até atingir o máximo de $12 \mathrm{~s}$. Respostas de seleção do estímulo de comparação correto, nas três tentativas do bloco, eram seguidas da apresentação do próximo bloco com o atraso ajustado em $1 \mathrm{~s}$, ou seja, o intervalo entre a remoção do estímulo modelo e a apresentação dos estímulos de comparação aumentava em 1s. Caso ocorresse uma ou mais respostas incorretas, era reapresentado o bloco anterior com diminuição de $1 \mathrm{~s}$ do atraso. Assim, erro em um bloco resultava na repetição de, pelo menos, dois blocos (o anterior, com menor atraso, e o bloco em que o erro ocorreu).

Em todos os treinos as respostas corretas eram seguidas por uma tela branca com a apresentação da figura de um smile (em português, carinha feliz) por $1,5 \mathrm{~s}$, concomitante com a apresentação de estímulos auditivos (e.g., aplausos, "Parabéns", "Muito bem!", “Ótimo"). As respostas incorretas eram seguidas da apresentação da figura de um "X" vermelho, por 1,5 s, também em uma tela branca. Logo após a apresentação das consequências para acerto ou erro, era apresentado o IET, que consistiu na apresentação de uma tela cinza por $1,5 \mathrm{~s}$, seguido por uma nova tentativa.

Tabela 1. Relações Treinadas, Quantidade de Blocos e Total de Tentativas Para Cada Treino e Teste das Duas Condições Experimentais do Experimento.

\begin{tabular}{|c|c|c|c|c|}
\hline Condição & Treino/Teste & Relação & Blocos & Tentativas \\
\hline \multirow{4}{*}{ Atraso Ajustado } & Treino AB & A1B1; A2B2; АЗB3. & 12 & 36 \\
\hline & Treino AC & $\mathrm{A} 1 \mathrm{C} 1 ; \mathrm{A} 2 \mathrm{C} 2 ;$ АЗС3. & 12 & 36 \\
\hline & $\begin{array}{l}\text { Treino misto } \\
\qquad \mathrm{AB} / \mathrm{AC}\end{array}$ & $\mathrm{A} 1 \mathrm{~B} 1 ; \mathrm{A} 2 \mathrm{~B} 2 ; \mathrm{A} 3 \mathrm{~B} 3 ; \mathrm{A} 1 \mathrm{C} 1 ; \mathrm{A} 2 \mathrm{C} 2 ; \mathrm{A} 3 \mathrm{C} 3$. & 12 & 72 \\
\hline & Testes & $\begin{array}{c}\mathrm{B} 1 \mathrm{~A} 1 ; \mathrm{B} 2 \mathrm{~A} 2 ; \mathrm{B} 3 \mathrm{~A} 3 ; \mathrm{C} 1 \mathrm{~A} 1 ; \mathrm{C} 2 \mathrm{~A} 2 ; \text { С } 3 \mathrm{~A} 3 ; \mathrm{B} 1 \mathrm{C} 1, \mathrm{~B} 2 \mathrm{C} 2 \\
\text { В3С3; C1B1, С2B2, В3С3. }\end{array}$ & 4 & 24 \\
\hline \multirow{4}{*}{ Atraso Fixo } & Treino DE & D1E1; D2E2; D3E3. & 12 & 36 \\
\hline & Treino DF & D1F1; D2F2; D3F3. & 12 & 36 \\
\hline & $\begin{array}{l}\text { Treino misto } \\
\text { DE/DF }\end{array}$ & D1E1; D2E2; D3E3; D1F1; D2F2; D3F3. & 12 & 72 \\
\hline & Testes & $\begin{array}{c}\text { E1D1; E2D2; E3D3; F1D1; F2D2; F3D3; E1F1, E2F2, } \\
\text { E3F3; F1E1, F2E2, E3F3. }\end{array}$ & 4 & 24 \\
\hline
\end{tabular}


Nos blocos de 1-11, em caso de erros em três blocos consecutivos, o treino era encerrado e reiniciado, o que poderia acontecer por, no máximo, três vezes. Para avançar de um treino para outro, era necessário atingir o critério de $100 \%$ de acerto na primeira exposição ao bloco 12. Se esse critério não fosse atingido em até três exposições, o participante era excluído do estudo.

Após os treinos das relações $\mathrm{AB}$ e AC, era realizado o treino misto composto por tentativas misturadas dessas duas relações. Inicialmente, todas as respostas produziam consequências diferenciais para acertos e erros e, posteriormente, apenas 50\% das respostas produziam consequências. O treino misto continha 12 blocos com seis tentativas (três para cada relação), era realizado com o procedimento de atraso ajustado, com critério para aumento e redução dos atrasos igual ao utilizado nos treinos simples. As dicas visuais utilizadas no primeiro bloco dos treinos simples não foram apresentadas durante o treino misto. Em caso de erros, eram utilizados os mesmos critérios de repetição e finalização dos blocos de 1-11 do treino simples, inclusive no bloco 12. Assim, era necessário que o participante atingisse o critério de $100 \%$ de acerto em todos os blocos para avançar para a etapa de teste de formação de classes de equivalência.

Treinos de relações condicionais da Condição Atraso Fixo. Nessa condição, os participantes foram expostos aos treinos simples (DE e DF) e misto com o procedimento de pareamento com atraso fixo. Em todas as tentativas, a resposta de observação sobre o estímulo modelo era seguida da remoção desse estímulo e, após um atraso fixo de 12 $\mathrm{s}$, eram apresentados os três estímulos de comparação. Respostas de seleção do estímulo de comparação correto eram seguidas da apresentação do próximo bloco de tentativas; e uma ou mais respostas incorretas resultavam na reexposição ao bloco de tentativas onde o (s) erro (s) ocorreu (ram). As demais características das tentativas de treino foram mantidas como especificado na descrição da Condição Atraso Ajustado.

Para manter constante o número de tentativas nas duas condições experimentais, a quantidade de blocos de cada treino da Condição Atraso Fixo foi definida para cada participante a partir da quantidade de blocos realizados na Condição Atraso
Ajustado. Nos treinos da Condição Atraso Fixo, o experimentador registrava o número de blocos realizadas e finalizava manualmente o treino assim que a quantidade de blocos fosse igual ao do mesmo treino da Condição Atraso Ajustado, previamente realizado.

Teste de formação de classes de equivalência. O teste das duas condições experimentais foi realizado com o procedimento de pareamento com atraso de $1 \mathrm{~s}$ e sem a programação de consequências diferenciais para respostas corretas e incorretas. Todas as respostas eram seguidas pela apresentação de uma tela cinza por 1,5 s (IET) e, posteriormente, por uma nova tentativa. O teste foi estruturado em quatro blocos de seis tentativas (Tabela 1). As relações simétricas BA (Condição Atraso Ajustado) ou ED (Condição Atraso Fixo) foram testadas no primeiro bloco; o teste das relações simétricas CA (Condição Atraso Ajustado) ou FD (Condição Atraso Fixo) foi realizado no segundo bloco de tentativas; no terceiro bloco foram testadas as relações de transitividade BC (Condição Atraso Ajustado) ou EF (Condição Atraso Fixo); e no quarto bloco foram testadas as relações de equivalência $\mathrm{CB}$ (Condição Atraso Ajustado) ou FE (Condição Atraso Fixo). Foi considerado como critério de formação de classes de equivalência, porcentagem de acerto igual ou superior a 83,3\% (ou 1 erro) em cada bloco de teste.

\section{Resultados}

A Figura 2 mostra a porcentagem de acerto nos testes de simetria, transitividade e equivalência para cada participante nas condições Atraso Ajustado e Atraso Fixo. Na Condição Atraso Ajustado, apenas P1 e P4 atingiram o critério de $100 \%$ de acerto em todos os testes realizados. P5 obteve $100 \%$ de acerto nos testes de transitividade e equivalência, mas nos testes de simetria o escore foi de 75\%. P3 também obteve 75\% de acerto nos testes de simetria desta condição, mas apenas $33,3 \%$ de acerto nos testes de transitividade e equivalência. $\mathrm{O}$ desempenho de $\mathrm{P} 2$ foi o mais baixo (33,3\% de acerto) em todos os testes dessa condição. Diferentemente, na Condição Atraso Fixo todos os participantes formaram classes de equivalência (escores nos testes igual ou superior a 83,3\% de acerto). 
P1

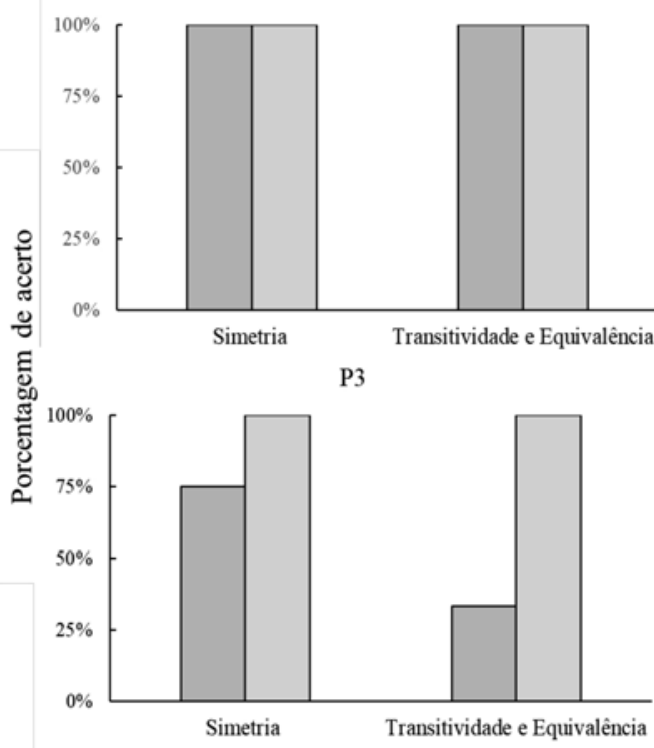

P2

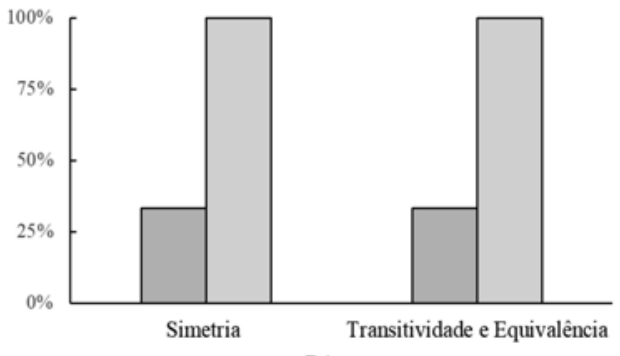

P4

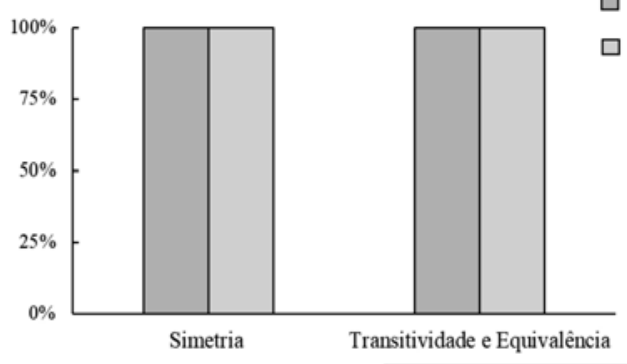

P5

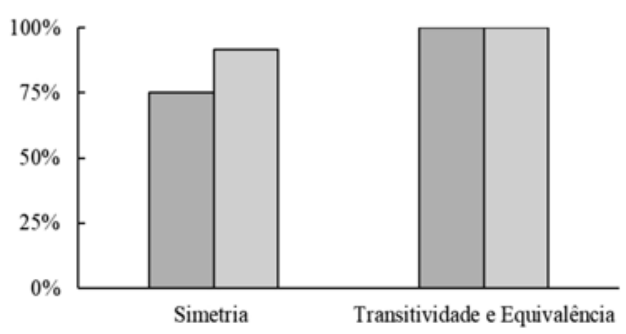

Figura 2. Porcentagem de acerto em todos os testes de formação de classe de equivalência nas Condições Atraso Fixo e Atraso Ajustado.

A Tabela 2 apresenta a quantidade de tentativas que cada participante realizou durante as etapas de treino simples e misto das duas condições experimentais, bem como o número de tentativas que foram programadas inicialmente e o número de erros em cada treino.

$\mathrm{Na}$ Condição Atraso Ajustado, apenas P4 atingiu o critério de $100 \%$ de acerto no Treino AB. Os demais participantes apresentaram de 3-13 erros e foram expostos a um número maior de tentativas, além da quantidade mínima (36), para atingir esse critério em todos os blocos. Os participantes P1, P2, P3 e P5 apresentaram erros durante a realização do bloco 1 do Treino AB, apesar da sinalização dos estímulos que deveriam ser relacionados. No Treino AC, somente P2 requereu mais do que 36 tentativas para atingir o critério de $100 \%$ de acer- to. $\mathrm{P} 1, \mathrm{P} 3, \mathrm{P} 4$ e $\mathrm{P} 5$ atingiram o critério na primeira exposição aos 12 blocos de tentativas do Treino AC. Com relação ao treino misto, todos os participantes atingiram o critério de $100 \%$ de acerto na primeira exposição. Na Condição Atraso Fixo, apenas P1 apresentou um erro no Treino DE. Os demais participantes atingiram o critério de 100\% de acerto na primeira exposição aos blocos de tentativas inicialmente programados em cada treino (simples e misto), de acordo com o acoplamento com a Condição Atraso Ajustado.

A partir da análise dos resultados dos treinos das condições Atraso Ajustado e Atraso Fixo (Tabela 2) e do desempenho nos testes de formação de classe de equivalência (Figura 3), verifica-se que desempenhos mais precisos nas tentativas de treino de relações condicionais foram acompa- 
nhados de desempenhos mais precisos nos testes de relações emergentes. Nos testes da Condição Atraso Ajustado, a porcentagem de acerto foi menor entre os participantes que emitiram uma maior quantidade de erros nas tentativas de treino (P2 e P3). Além disso, na Condição Atraso Fixo todos os participantes responderam de acordo com o critério definido para a formação de classes de equivalência. Nesta condição todos os participantes, com exceção de P1, completaram os treinos simples e misto com $100 \%$ de acerto.

Tabela 2. Quantidade de Tentativas Realizadas, Número Mínimo de Tentativas Programadas e Número de Erros nas Tentativas de Treino para cada Participante nas Condições Atraso Ajustado e Atraso Fixo.

\begin{tabular}{|c|c|c|c|c|c|c|}
\hline \multirow[t]{2}{*}{ Condição } & \multirow[t]{2}{*}{ Treino } & \multicolumn{5}{|c|}{ Participante } \\
\hline & & P1 & P2 & P3 & P4 & P5 \\
\hline \multirow{3}{*}{$\begin{array}{l}\text { Atraso } \\
\text { Ajustado }\end{array}$} & Treino AB & 45/36 (3) & $72 / 36(13)$ & 63/36 (8) & $36 / 36$ & $48 / 36(4)$ \\
\hline & Treino AC & $36 / 36$ & $42 / 36(1)$ & $36 / 36$ & $36 / 36$ & $36 / 36$ \\
\hline & $\begin{array}{l}\text { Treino misto } A B / \\
A C\end{array}$ & $72 / 72$ & $72 / 72$ & $72 / 72$ & $72 / 72$ & $72 / 72$ \\
\hline \multirow{3}{*}{ Atraso Fixo } & Treino DE & $45 / 45$ (1) & $72 / 72$ & $63 / 63$ & $36 / 36$ & $48 / 48$ \\
\hline & Treino DF & $36 / 36$ & $42 / 42$ & $36 / 36$ & $36 / 36$ & $36 / 36$ \\
\hline & $\begin{array}{l}\text { Treino misto DE/ } \\
\text { DF }\end{array}$ & $72 / 72$ & $72 / 72$ & $72 / 72$ & $72 / 72$ & $72 / 72$ \\
\hline
\end{tabular}

Nota. O número à esquerda da barra refere-se ao número de tentativas realizadas pelo participante; o número à direita da barra refere-se ao número mínimo de tentativas programadas; o número entre parêntese refere-se ao número de erros.

\section{Discussão}

O objetivo do presente experimento foi investigar o efeito do tipo do atraso (fixo ou ajustado) nos treinos com tarefas de pareamento ao modelo na formação de classes de equivalência. $\mathrm{Na}$ Condição Atraso Ajustado, foi verificado que, dentre os cinco participantes, apenas dois (P1 e P4) atingiram o critério de formação de classe de equivalência, sendo que P5 apresentou escore superior a 83,3\% de acerto, somente nos testes das relações de transitividade e equivalência. Na Condição Atraso Fixo, todos os participantes tiveram 100\% de acerto nos testes de formação de classe de equivalência. Esses resultados estão coerentes com os que foram obtidos por Lian e Arntzen (2011), que também demonstraram melhores desempenhos nos testes após a manipulação do atraso fixo nos treinos de relações condicionais.

Entretanto, os resultados obtidos no presente estudo referem-se à uma manipulação de $12 \mathrm{~s}$ do atraso fixo e $12 \mathrm{~s}$ de atraso ajustado em um delineamento intrassujeito com estudantes universitários. Diferentemente, o estudo de Lian e Arntzen 
(2011) foi realizado com crianças e foi utilizado o delineamento entre sujeitos tanto ao manipular a duração do atraso quanto ao manipular o tipo de atraso (fixo ou ajustado). Lian e Arntzen (2011) verificaram melhores desempenhos quando o treino de relações condicionais foi realizado com o atraso máximo de $3 \mathrm{~s}$, tanto no pareamento com atraso ajustado como no pareamento com atraso fixo. No presente experimento, dois participantes da Condição Atraso Ajustado e todos os participantes (5) da Condição Atraso Fixo formaram classes de equivalência, mesmo com o atraso máximo de 12 s. Esse resultado diverge dos que foram obtidos por Lian e Arntzen (2011) que verificaram formação de classes de equivalência para mais participantes expostos ao menor atraso (3s). Entretanto, corrobora com o estudo de Vaidya e Smith (2006) que verificou melhores desempenhos nos testes de simetria com maiores atrasos ( $8 \mathrm{~s}$ ), em comparação com os atrasos de 0 e 2 s; com Arntzen et al. (2007) que demonstraram formação de classe de equivalência com longos atrasos (12 s); e Lian e Arntzen (2013) que verificaram precisão do responder por identidade mesmo quando o atraso foi de 12,25 s.

Os resultados do presente estudo e de Lian e Arntzen (2011) sugerem que, em relação a duração do atraso, a idade dos participantes é um aspecto que deve ser considerado. Ferraro et al. (1971) expôs crianças de diferentes faixas etárias a um procedimento de pareamento de identidade de cores com atraso ajustado e foi verificada aprendizagem das relações ensinadas com atraso médio de $26,4 \mathrm{~s}$ para crianças mais velhas (121 a 130 meses) e atraso médio de apenas $1,3 \mathrm{~s}$ para crianças de uma faixa etária menor (51 a 60 meses). Com crianças de 6-10 anos (72 a 120 meses), após o treino de discriminações condicionais com estímulos abstratos, Lian e Arntzen (2011) verificaram formação de classes de equivalência para mais participantes com atrasos menores (3s). A diferença entre os desempenhos das crianças da mesma faixa etária nesses dois estudos pode estar relacionada com o tipo de tare$\mathrm{fa}$ (identidade de cores $\mathrm{x}$ pareamento com figuras abstratas). No presente estudo, com tarefa similar à de Lian e Arntzem (2011), os participantes adultos formaram classes com atraso maior (12 s) do que as crianças, enquanto no estudo de Albrecht (2019) adultos aprenderam identidade entre figuras abs- tratas com atrasos de $20 \mathrm{~s}$.

Os sujeitos P2, P3 e P5 apresentam um melhor desempenho durante os testes de formação de classe de equivalência da Condição Atraso Fixo quando comparado com os resultados obtidos durante a Condição Atraso Ajustado. Esse desempenho pode ser considerado um efeito de Learning set, comportamento de ordem superior que demonstra como a aprendizagem de novas relações entre estímulos depende das relações aprendidas anteriormente (Catania, 1999). Alguns aspectos do procedimento de pareamento ao modelo com atraso podem ter favorecido esse tipo de aprendizagem, como a exposição prolongada à tarefa de pareamento ao modelo, comportamentos privados, como nomeação, que podem ter sido reforçados a partir das consequências diretas apresentadas durante o treino de discriminações condicionais, e similaridades entre as tentativas de treino e de teste, apesar dos diferentes atrasos manipulados nos treinos. Para que esse efeito fosse controlado seria necessário balancear a ordem de apresentação das condições experimentais entre os participantes. Entretanto, no presente estudo foi priorizado o controle do número de tentativas a que cada participante foi exposto nas duas condições. Se o balanceamento na ordem de exposição às condições experimentais fosse realizado, não seria possível controlar o número de tentativas para os participantes expostos à ordem Condição Atraso Fixo seguida por Condição Atraso Ajustado, pois, por características específicas do ajuste do atraso em função do desempenho dos participantes, acoplar as tentativas desta condição de acordo com a quantidade de tentativas realizadas na Condição Atraso Fixo prejudicaria o ajuste da duração final do atraso, a quantidade de tentativas que cada participante realizaria em cada bloco da Condição Atraso Ajustado e, consequentemente, o controle de tais variáveis. Por esse motivo o balanceamento da ordem de exposição às condições experimentais entre os sujeitos não foi possível de ser realizado. Novas pesquisas poderão investigar o efeito dessa aprendizagem e verificar se, ao controlar a ordem de exposição às condições com diferentes manipulações do atraso, diferentes resultados seriam observados, como os demonstrados por P2, P3 e P5.

Arntzen, Nartey e Fields (2018) realizou um es- 
tudo para verificar o efeito do atraso com 100 participantes, organizados em 10 grupos. Cinco grupos foram expostos a um treino prévio de relações condicionais com estímulos arbitrários (CX). Os demais foram expostos a um treino prévio de relações condicionais de identidade (CC). Nessa etapa, cada um dos cinco grupos foi exposto a um valor de atraso fixo que variou entre 0 s, 1 s, 3 s, 6 s e 9 s. Adicionalmente, 20 participantes foram alocados em dois grupos (PIC e ABS) e foram expostos diretamente à etapa de linha de base, com pareamento simultâneo, sem exposição ao treino prévio. A linha de base consistiu no treino das relações $\mathrm{AB}, \mathrm{BC}, \mathrm{CD}$ e DE. Para o grupo PIC o conjunto C era composto por estímulos familiares. Para os demais, incluindo os grupos que foram expostos ao treino prévio de identidade, todas as relações foram compostas por estímulos abstratos. Foi verificado que o desempenho nos testes de formação de classes de equivalência com pareamento simultâneo foi mais preciso para os participantes expostos ao treino prévio de relações condicionais com atraso e para o grupo PIC. Esse resultado aponta para a importância de se estabelecer previamente comportamentos elementares que contribuirão para o estabelecimento posterior de desempenhos mais complexos. Sugere-se que pesquisas futuras investiguem o efeito de treinos prévios de relações condicionais antes de expor os participantes à tarefa de pareamento ao modelo com atraso fixo ou ajustado.

A utilização nos treinos das dicas "Na presença deste" acima dos estímulos modelo e "Escolha este" acima dos estímulos de comparação no primeiro bloco de tentativas baseou-se no estudo de Bortolotti e de Rose (2012). Estas dicas foram utilizadas para minimizar os erros e para igualar todos os blocos de tentativas dos treinos das relações simples $\mathrm{AB}$ e $\mathrm{AC}$ na Condição Atraso Ajustado e DE e DF na Condição Atraso Fixo. Entretanto, a maioria dos erros apresentados na Condição Atraso Ajustado ocorreu no primeiro bloco de tentativas, mesmo com a utilização dessas dicas. Um fator que pode ter contribuído refere-se à apresentação dos três estímulos de comparação (estímulos S+e $\mathrm{S}-$ ) desde a primeira tentativa de treino, além da possibilidade de controle por características dos estímulos ou da tarefa não identificados pelo experimentador. Para minimizar a ocorrência de er- ros no contato inicial do participante com a tarefa, uma alternativa seria a utilização do procedimento de esvanecimento, procedimento que minimiza a ocorrência de erros (Catania, 1999), iniciando a primeira tentativa com a apresentação do estímulo de comparação correlacionado com reforço e aumentando gradualmente a quantidade de estímulos de comparação.

Apesar das limitações metodológicas apontadas, os resultados encontrados contribuem para a literatura sobre controle de estímulos, principalmente para a área de equivalência de estímulos, ao avançar nas investigações acerca do tipo de manipulação do atraso, uma vez que os resultados mostram vantagem do atraso fixo, em relação ao atraso ajustado, sobre o desempenho emergente em participantes adultos. Entretanto, os resultados acerca dos efeitos do pareamento com atraso sobre o responder emergente no presente estudo ainda não são conclusivos. É necessário que futuros estudos busquem elucidar melhor as variáveis envolvidas no procedimento de pareamento ao modelo com atraso fixo ou ajustado através de investigações paramétricas acerca da duração do atraso, da ordem de exposição aos atrasos, ao tipo de atraso manipulado, à idade e condições neurológicas dos participantes.

\section{Referências}

Albrecht, C. (2019). Efeitos de estímulos visuais e auditivos sobre o desempenho em uma tarefa de matching de identidade com ajuste do atraso [Dissertação de Mestrado, Universidade de Brasília]. Repositório Institucional da Universidade de Brasília https://repositorio. unb.br/handle/10482/36829

Aggio, N. M., \& Domeniconi, C. (2012). Formação e manutenção de classes de estímulos equivalentes: Um estudo com participantes da terceira idade. Acta Comportamentalia, 20(1), 29-43. Recuperado de http://pepsic.bvsalud.org/pdf/ actac/v20n1/a04.pdf

Albuquerque, A. R., \& Melo, R. M. (2005). Equivalência de estímulos: Conceito, implicações e possibilidades de aplicação. Em J. Abreu- 
Rodrigues \& M. R. Rodrigues (Orgs.). Análise do comportamento: Pesquisa, teoria e aplicação (pp. 245-264). Porto Alegre: Artmed.

Arntzen, E. (2006). Delayed matching to sample: Probability of responding in accord with equivalence as a function of different delays. The Psychological Record, 56, 135-167. doi:10.1007/ bf03395541

Arntzen, E. (2012). Training and testing parameters in formation of stimulus equivalence: Methodological issues. European Journal of Behavior Analysis, 13(1), 123-135. doi:10.1080 /15021149.2012.11434412

Arntzen, E., Galaen, T., \& Halvorsen, L. R. (2007). Different retention intervals in delayed matching-to-sample: Effects of responding in accord with equivalence. European Journal of Behavior Analysis, 8, 177-191. doi:10.1080/15021149.20 07.11434281

Arntzen, E., Nartey, R. K., \& Fields, L. (2018). Graded delay, enhanced equivalence class formation, and meaning. The Psychological Record, 68 (2), 123-140. doi:10.1007/s40732-018-02716

Arntzen, E., \& Steingrimsdottir, H. S. (2014). On the use of variations in a delayed matching-tosample procedure in a patient with neurocognitive disorder. In M. H. Swahn, J. B. Palmier, \& S. M. Braunstein (Eds.), Mental disorder (pp. 123-138). iConcept Press Ltd.

Arntzen, E., Steingrimsdottir, H. S., \& Antonsen, A. B. (2013). Atferdsmessige studier av demens: Effekten av ulike varianter av matching-to-sample prosedyrer. Norsk Tidsskrift for Atferdsanalyse, 40(1), 17-29. Recuperado de http://hdl.handle.net/10642/1760

Bortoloti, R., \& de Rose, J. C. (2012). Equivalent stimuli are more strongly related after training with delayed matching than after simultaneous matching: A study using the implicit relational assessment procedure (IRAP). The Psychological Record, 62, 41-54. doi:10.1007/ bf03395785

Carr, D., Wilkinson, K. M, Blackman, D., \& McIlvane, W. (2000). Equivalence classes in individual with minimal verbal repertoires. Journal of the Experimental Analysis of Behavior, 74(1), 101-114. doi:10.1901/jeab.2000.74-101
Catania, A. C. (1999). Aprendizagem: Comportamento, linguagem e cognição, $4^{\mathrm{a}} \mathrm{ed}$. Porto Alegre: Artmed.

de Rose, J. C., de Sousa, D. G., \& Hanna, E. S. (1996). Teaching reading and spelling: Exclusion and stimulus equivalence. Journal of Applied Behavior Analysis, 29(4), 451-469. doi:10.1901/ jaba.1996.29-451

Ferraro, D. P., Francis, E. W., \& Perkins, J. J. (1971). Titrating delayed matching to sample in children. Developmental Psychology, 5(3), 488-493. doi: 10.1037/h0031598

Garcia, Y. A., \& Rehfeldt, R. A. (2008). The effects of common names and common FR responses on the emergence of stimulus equivalence classes. European Journal of Behavior Analysis, 9(2), 99-120. doi:10.1080/15021149.2008.11434298

Hanna, E. S., Batitucci, L. A. V., \& Batitucci, J. S. L. (2014). Software Contingência Programada: Utilidade e funcionalidades. Revista Brasileira de Análise do Comportamento, 10, 97-104. doi: http://dx.doi.org/10.18542/rebac.v10i1.3949

Haydu, V. B., \& Morais, L. P. (2009). Formação, manutenção e recuperação de relações equivalentes em adultos da terceira idade. Psicologia Argumento, 27(59), 323-336. doi: 10.7213/rpa. v27i59.19855

Laporte, F. F., \& Melo, R. M. (2016). Seguimento de instruções e repertório recombinativo: Efeito da formação de classes de equivalência e do tipo de composição dos estímulos. Acta comportamentalia, 24(3), 297-313. Recuperado de http:// www.revistas.unam.mx/index.php/acom/article/view/56965

Lian, T., \& Arntzen, E. (2011). Training conditional discriminations with fixed and titrated delayed matching-to-sample in children. European Journal of Behavior Analysis, 12(1), 173-193. doi: 10.1080/15021149.2011.11434362

Lian, T., \& Arntzen, E. (2013). Delayed matchingto-sample and linear series training structures. The Psychological Record, 63, 545-562. doi: 10.11133/j.tpr2013.63.3.010

Melo, R. M., \& Serejo, P. (2009). Equivalência de estímulos e estratégias de intervenção para crianças com dificuldade de aprendizagem. Interação em Psicologia, 13(1), 103-112. doi: http://dx.doi. org/10.5380/psi.v13i1.8723 
Nalini, L. E. G. (2002). Determinação empírica da nomeabilidade de estímulos: implicações para o estudo da relação de nomeação [Tese de Doutorado não publicada]. Universidade de Brasília.

Oliveira, J. M. (2014). Mudanças no treino discriminativo de pseudopalavras e seus efeitos sobre a observação dos estímulos e o controle pelas letras [Dissertação de Mestrado não publicada]. Universidade de Brasília.

Saunders, R. R., Chaney, L., \& Marquis, J. G. (2005). Equivalence class establishment two, three, and four choice matching to sample by senior citizens. The Psychological Record, 55(4), 539-559. doi:10.1007/bf03395526

Sidman, M., \& Cresson, O. (1973). Reading and crossmodal transfer of stimulus equivalences in severe retardation. American Journal of Mental Deficiency, 77(5), 515-523. Recuperado de https://www.ncbi.nlm.nih.gov/ pubmed/4267398

Sidman, M., \& Tailby, W. (1982). Conditional discrimination vs. matching to sample: an expansion of the testing paradigm. Journal of the Experimental Analysis of Behavior, 37, 5-22. doi: 10.1901/jeab.1982.37-5

Sidman, M., Willson-Morris, M., \& Kirk, B. (1986). Matching-to-sample procedures and the equivalence relations: The role of naming. Analysis and intervention in developmental disabilities, 6 , 1-19. doi: 10.1016/0270-4684(86)90003-0

Vaidya, M., \& Smith, K. N. (2006). Brief report: Delayed matching-to-sample training facilitates derived relational responding. Experimental Analysis of Human Behavior Bulletin, 24, 9-16.

Wilson, K. G., \& Hayes, S. C. (1996). Resurgence of derived stimulus relations. Journal of The Experimental Analysis of Behavior, 66(3), 267281. doi: 10.1901/jeab.1996.66-267

Wulfert, E., \& Hayes, S. C. (1988). Transfer of a conditional ordering response through conditional equivalence classes. Journal of the Experimental Analysis of Behavior, 50,125-144. doi: 10.1901/ jeab.1988.50-125

\section{Informações do Artigo}

Histórico do artigo:

Recebido em: 18/09/2019

Primeira decisão editorial: 21/07/2020

Segunda decisão editorial: 13/10/2020

Aceito em: 21/10/2020

Editor associado: Marcelo Silveira 